\title{
DÜBLIN
}

Technological University Dublin ARROW@TU Dublin

\section{Germanium Microsphere High-Q Resonator}

\author{
Pengfei Wang \\ Technological University Dublin, pengfei.wang@tudublin.ie \\ Timothy Lee \\ University of Southampton \\ Ming Ding \\ University of Southampton
}

See next page for additional authors

Follow this and additional works at: https://arrow.tudublin.ie/engscheceart

Part of the Systems and Communications Commons

\section{Recommended Citation}

Wang, P. et al: Germanium microsphere high-Q resonator. Optics Letters, Vol. 37, 4, 2012, pp.728-730. doi;10.1364/OL.37.000728

This Article is brought to you for free and open access by the School of Electrical and Electronic Engineering at ARROW@TU Dublin. It has been accepted for inclusion in Articles by an authorized administrator of ARROW@TU Dublin. For more information, please contact arrow.admin@tudublin.ie, aisling.coyne@tudublin.ie, gerard.connolly@tudublin.ie.

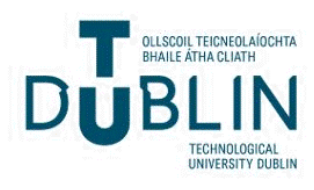




\section{Authors}

Pengfei Wang, Timothy Lee, Ming Ding, Anirban Dhar, Thomas Hawkins, Paul Foy, Yuliya Semenova, Qiang Wu, Jayanta Sahu, Gerald Farrell, John Ballato, and Gilberto Brambilla 


\title{
Germanium microsphere high- $Q$ resonator
}

\author{
Pengfei Wang, ${ }^{1,2, *}$ Timothy Lee, ${ }^{1}$ Ming Ding, ${ }^{1}$ Anirban Dhar,,${ }^{1}$ Thomas Hawkins, ${ }^{3}$ Paul Foy, ${ }^{3}$ Yuliya Semenova, ${ }^{2}$ \\ Qiang Wu, ${ }^{2}$ Jayanta Sahu, ${ }^{1}$ Gerald Farrell, ${ }^{2}$ John Ballato, ${ }^{3}$ and Gilberto Brambilla ${ }^{1}$ \\ ${ }^{1}$ Optoelectronics Research Centre, University of Southampton, Southampton SO17 1BJ, UK \\ ${ }^{2}$ Photonics Research Centre, Dublin Institute of Technology, Kevin Street, Dublin 8, Ireland \\ ${ }^{3}$ Center for Optical Materials Science and Engineering Technologies (COMSET), \\ Clemson University, Clemson, South Carolina 29634 USA \\ *Corresponding author: pw3y09@orc.soton.ac.uk
}

Received November 18, 2011; revised January 11, 2012; accepted January 12, 2012; posted January 13, 2012 (Doc. ID 158063); published February 15, 2012

\begin{abstract}
In this Letter, the fabrication and characterization of a microsphere resonator from the semiconductor germanium is demonstrated. Whispering gallery modes are excited in a $46 \mu \mathrm{m}$ diameter germanium microsphere resonator using evanescent coupling from a tapered silica optical fiber with a waist diameter of $2 \mu \mathrm{m}$. Resonances with $Q$ factors as high as $3.8 \times 10^{4}$ at wavelengths near $2 \mu \mathrm{m}$ are observed. Because of their ultrahigh optical nonlinearities and extremely broad transparency window, germanium microsphere resonators offer the potential for optical processing devices, in particular at long wavelengths, such as around $2 \mu \mathrm{m}$. (C) 2012 Optical Society of America

OCIS Codes: $130.5990,140.3510,140.4780,160.6000$.
\end{abstract}

A variety of well-known semiconductor materials have been developed as the foundation of modern microelectronics and microphotonics. As a semiconductor material for microphotonics applications [1] $]$, germanium is particularly attractive owing to its large nonlinearity (thousands of times that of conventional silica), high optical damage threshold compared with traditional nonlinear glass materials, and, above all, its extremely broad transparency window that extends from the near-IR well into the mid-IR wavelength $(\lambda=2-15 \mu \mathrm{m})$ spectral region. Ge-based optical components have found numerous applications in IR imaging systems operating in the atmospheric windows (3-5 and 8-14 $\mu \mathrm{m})$, where the principal natural greenhouse gases do not exhibit strong absorption. Ge also is a good electromagnetic shielding material, an attribute that has become increasingly important for modern military applications, where other signals (within the millimeter and centimeter wavelength range) can be strong enough to interfere with nearby IR systems. However, to date, this material has not been exploited for high- $Q$ resonators.

Over the past few decades, microsphere high- $Q$ resonators have increasingly attracted interest because they add a range of significant functionalities to active and passive photonic devices, such as feedback, wavelength selectivity, energy storage to allow dispersion control, enhanced nonlinearity, resonant filtering, and ultralow threshold lasing [2]. In particular, Ge has been used as dopant of silica microspheres to study frequency trimming and photonic filters [3,4]. Photonic devices based on optical microresonators that strongly confine photons form the basis for developing compact-size, low-power, and high-speed photonic circuits.

Recently, semiconductor microresonators have shown potential for achieving three-dimensional optoelectronic integration in wavelength division multiplexing systems [5]. For example, a channel-dropping filter with a silicon microsphere using a fiber taper has been developed [ [6], and optical resonances from a silicon microcylindrical resonator have been observed at the wavelength $\lambda \sim$
$1.55 \mu \mathrm{m}$ within the conventional band (C-band) used for near-IR telecommunications [7]. Ge has attracted growing attention as a possible complementary metal-oxide semiconductor compatible light emitter that can be easily integrated onchip, and resonance modes have been observed over the range of $1500-1675 \mathrm{~nm}$ for a Ge resonator diode on silicon fabricated by integrating a germanium LED with a microdisk cavity [8]. Yet, Ge has a strong absorption at those wavelengths, thus $Q$ factors tend to be extremely small. On the contrary, for $\lambda>1.8 \mu \mathrm{m} \mathrm{Ge}$ absorption becomes a minor issue. The range around $\lambda=$ $2 \mu \mathrm{m}$ is particularly important because of its many applications, such as gas detection, long-range lidar for heterodyne measurement, and optical pumping of longer wavelength solid-state lasers. Free-space optical communication has also assumed increased importance because of the 2-2.5 $\mu \mathrm{m}$ atmospheric transparency window [9]. For these reasons, in the following experimental investigation, the optical resonance properties of a Ge microsphere resonator are characterized at $\lambda \sim 2 \mu \mathrm{m}$. In this Letter, resonant modes with $Q$ factors up to $3.8 \times 10^{4}$ are observed at $\lambda \sim 2 \mu \mathrm{m}$ for a Ge microsphere resonator fabricated from a Ge-core optical fiber using both a $\mathrm{CO}_{2}$ laser and chemical etching.

Ge microspheres were fabricated by exposing a glassclad Ge-core optical fiber to a $30 \mathrm{~W} \mathrm{CO}_{2}$ laser (Synrad, USA) beam $(\lambda=10.6 \mu \mathrm{m})$, which was focused using a cylindrical $\mathrm{ZnSe}$ lens. The fiber was manufactured at Clemson University (USA) and had core and cladding diameters of 15 and $150 \mu \mathrm{m}$, respectively [10]. The Ge core was positioned out of the focal point of the laser in order to achieve a wide heating region and an appropriate temperature between the melting point of $\mathrm{Ge}\left(938^{\circ} \mathrm{C}\right)$ and the softening point of the borosilicate glass cladding $\left(>1260^{\circ} \mathrm{C}\right)$. Because of the low viscosity, surface tension shapes the molten Ge in the form of a Ge spheres grown inside the fiber cladding, with the additional benefit of a surface smoothness typical of glass surfaces. The typical diameter of the manufactured spheres attached to Ge fiber stems was 40 to $50 \mu \mathrm{m}$. A microscope photograph of a 
typical Ge microsphere embedded in the borosilicate glass cladding is shown in Fig. 1(a). The Ge resonator was released by first etching the large majority of glass cladding away using a $48 \%$ hydrofluoric acid (HF), and then immersing the Ge sphere into a mixed solution consisting of buffered ammonium fluoride and HF solution (volume ratio 20:1) for 5-10 min for the final etching. Microspheres then were rinsed in deionized water for cleaning any residual HF. As shown in Fig. 1(b), no inhomogeneity or gross surface roughness could be observed on the microsphere surface.

The spectral response of the Ge microsphere was investigated using the experimental apparatus shown in Fig. 2. A broadband source in the range $\lambda=1.8-2.2 \mu \mathrm{m}$ was built using the amplified spontaneous emission of a Tm-doped fiber pumped at $\lambda=1.55 \mu \mathrm{m}$. Light from a tunable laser delivering a power of $P=1 \mathrm{~mW}$ at $\lambda=1.55 \mu \mathrm{m}$ was launched into an $\mathrm{Er}^{3+}$-doped fiber amplifier, which amplified the output signal to $P=100 \mathrm{~mW}$. In order to avoid damage to the laser source and to other optical components by backreflections, an isolator at $\lambda=1.55 \mu \mathrm{m}$ was used between the amplifier module and the Tm-doped fiber; the single-mode fiber also was wrapped on a mandrel at a bend radius of $18 \mathrm{~mm}$ with 10 turns to absorb possible backreflections at long wavelengths. The output of the coiled single-mode fiber was spliced to a 4-m-long section of a Tm-doped fiber. The Tm-doped fiber was made at the Optoelectronics Research Centre (Southampton, UK) and it had alumino-silicate core with a diameter of $10 \mu \mathrm{m}$ and NA of 0.17 . A relatively high $\mathrm{Tm}^{3+}$ concentration $(\sim 1$ wt.\%) was used to achieve efficient absorption of pump light in a short length of fiber. The $1550 \mathrm{~nm}$ pump laser radiation excites $\mathrm{Tm}^{3+}$ ions into the ${ }^{3} F_{4}$ level through ground-state absorption. The transition ${ }^{3} F_{4} \rightarrow$ ${ }^{3} H_{6}$ is responsible for the laser emission near $\lambda \sim 2 \mu \mathrm{m}$. The output at maximum gain was $\sim-30 \mathrm{dBm}$ at $\lambda=$ $1.9 \mu \mathrm{m}$ and was limited by the pump power and the absorption of the $\mathrm{Tm}^{3+}$-doped fiber. Figure 3 shows the emission spectrum as measured by a Yokogawa AQ6375 optical spectrum analyzer (OSA) (wavelength range, $1200-2400 \mathrm{~nm}$ ).

The output of the Tm-doped fiber is fusion spliced to a short silica fiber containing a tapered microfiber section with a diameter of $2 \mu \mathrm{m}$, which is used for evanescent coupling to the Ge microsphere. The output signal is analyzed using the long wavelength OSA. The separation between the microsphere and the tapered silica fiber was controlled with a precision nanotranslation stage equipped with piezoelectric actuators and stepper motors and mon-

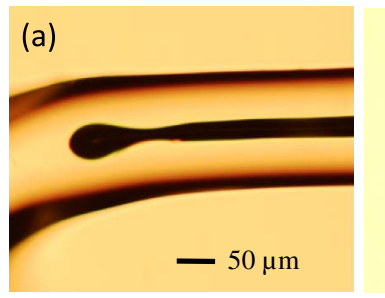

(b)

Fig. 1. (Color online) Microscope images of (a) germaniumcore fiber after $\mathrm{CO}_{2}$ laser heating and (b) germanium microresonator after the borosilicate cladding was removed by buffered HF. The resonator had a diameter of $46 \mu \mathrm{m}$ and was connected to a stem to facilitate handling.

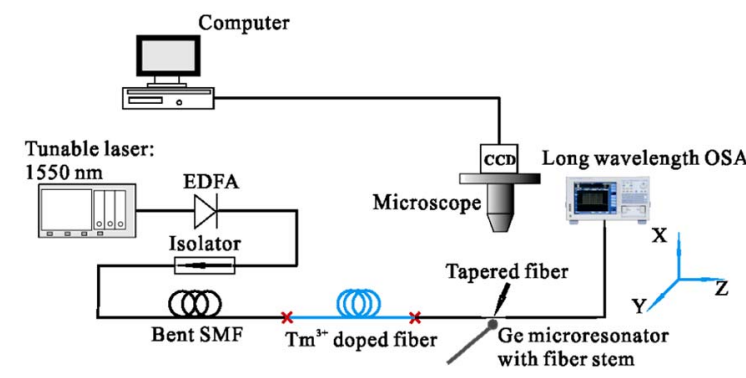

Fig. 2. (Color online) Experimental apparatus used for microsphere resonance characterization.

itored using a microscope equipped with a CCD camera. The Ge fiber stem supporting the Ge microresonator ensured that the resonator orientation remained fixed with respect to the tapered silica coupling fiber as it was translated across and away from it.

Figure 4(a) (top) shows the resonance spectra of the Ge microsphere over the wavelength range 1975$2025 \mathrm{~nm}$. The tapered fiber excitation clearly produces dense spectral features, as already observed with other high-index glass microspheres [11]. This is due to the excitation of many higher-order radial modes by the low effective index fiber taper and many nondegenerate higher-order angular modes associated with microsphere ellipticity [12]. The effective index of the fundamental mode in a $2 \mu \mathrm{m}$ tapered fiber at $\lambda \sim 2 \mu \mathrm{m}$ is approximately $n_{\text {eff }} \sim 1.35$, which represents a large difference compared to the effective index of the Ge microresonator. For this reason, the taper will not excite a single mode in the microsphere, but will excite a large number of modes, as shown in Fig. 4. In this study, a tapered silica fiber (instead of a phase-matched tapered high-index fiber) was used to excite whispering gallery modes (WGMs), mainly to minimize any nonlinear effects from the delivery fiber. The $Q$ of a microsphere resonator can be easily estimated from its WGM spectrum through the relation $Q=\lambda / \Delta \lambda$, where $\Delta \lambda$ is the FWHM and $\lambda$ is the central wavelength of the resonance. Figure 4 (a) (bottom) presents the spectrum over a narrow wavelength range, showing the high- $Q$ nature of the observed resonance dips. Because of the limited OSA resolution (50 pm), the measured resonance FWHM $(0.0526 \mathrm{~nm})$ is an overestimation, resulting in an underestimated $Q$ factor of $3.8 \times 10^{4}$. Figure $4(\mathrm{~b})$ shows that approximately $36 \%$ of

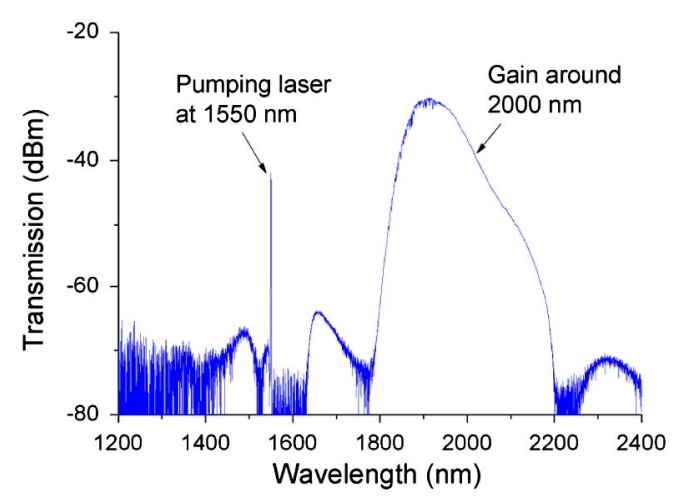

Fig. 3. (Color online) Emission spectrum of the ${ }^{3} F_{4} \rightarrow{ }^{3} H_{6}$ emission (1.8-2.2 $\mu \mathrm{m}$ ) from a 4-m-long thulium-doped fiber pumped by a $1550 \mathrm{~nm}$ laser. 

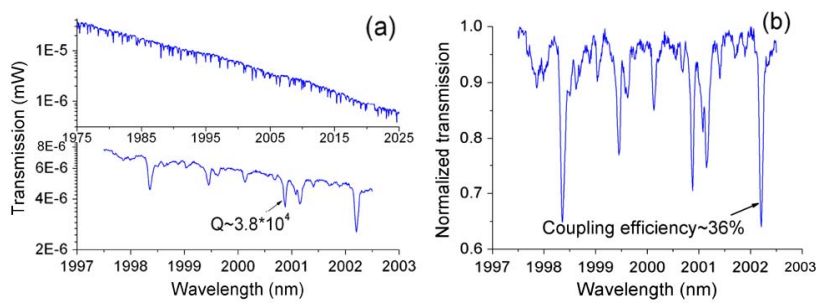

Fig. 4. (Color online) (a) Top: experimental resonance spectrum for wavelengths between 1975 and $2025 \mathrm{~nm}$ for the microsphere with a diameter of $46 \mu \mathrm{m}$. Bottom: experimental resonance spectrum in the region of $1997.5-2002.5 \mathrm{~nm}$. (b) Normalized transmission as a function of wavelength.

the light in the tapered fiber is coupled to the microsphere at resonance.

To illustrate the coupling issues between WGMs within the Ge microsphere and the mode in the taper, an analysis of the microsphere resonator excitation was carried out based on the comprehensive theoretical investigation presented in [13]. The refractive index of the tapered fiber was taken to be 1.438 , while the refractive index of the Ge microsphere was taken to be 4.104 at a wavelength of $2 \mu \mathrm{m}$. The higher-order (high $n$ ) radial modes (and also higher-order Hermite-Gaussian modes with $m<l$ ) have an $n_{\text {eff }}$ closer to that of the mode propagating in the fiber taper than the fundamental WGM mode $(n=1)$, thus they can be excited more efficiently than the fundamental WGM. In fact, coupling efficiency is strongly related to the phase mismatch; thus, modes that have smaller phase mismatch experience stronger coupling. In the simulations carried out to evaluate $n_{\text {eff }}$ of the Ge microsphere WGMs, the value of $l(l=m)$ for each point was chosen to keep the resonant wavelength close to $2 \mu \mathrm{m}$. As shown in Fig. 5, the higher-order modes $(n \gg 2)$ have lower $l$ and $m$ values and, hence, a lower $n_{\text {eff }}$. Furthermore, the resonance spectrum is likely to be complicated by the nondegeneracy of the aforementioned Hermite-Gaussian modes with different order $m$, due to the ellipticity observed in fabricated microspheres.

Note that, compared to equivalent silica microspheres, the index contrast between the Ge sphere and air is $\sim 7$ times greater. For this reason, the WGMs in a Ge sphere experience stronger confinement, which reduces modal overlap with the taper mode.

In conclusion, the fabrication of Ge microspheres has been demonstrated. WGM resonances excited by evanescent coupling from a silica fiber taper with a diameter $d \sim$ $2 \mu \mathrm{m}$ have been observed and a high- $Q$ factor up to $3.8 \times 10^{4}$ was recorded at $\lambda \sim 2 \mu \mathrm{m}$. Compared with conventional silica and other semiconductor materials, such as GaAs and $\mathrm{Si}, \mathrm{Ge}$ has an ultrahigh nonlinearity and comparable low absorption in an ultrabroad wavelength range [14], which covers the near- and mid-IR military communication windows; in this region, Ge microspheres could be used for all-optical switching and data processing. Moreover, it is anticipated that such a $\mathrm{Ge}$ microsphere resonator could also be utilized as the basis for novel implementations of microphotonics devices, including highly integrated optical switches, electro-optical time delay modulators, quantum well microdevices, and Raman scattering microlasers: such theoretical and experimental nonlinear characterization is underway.

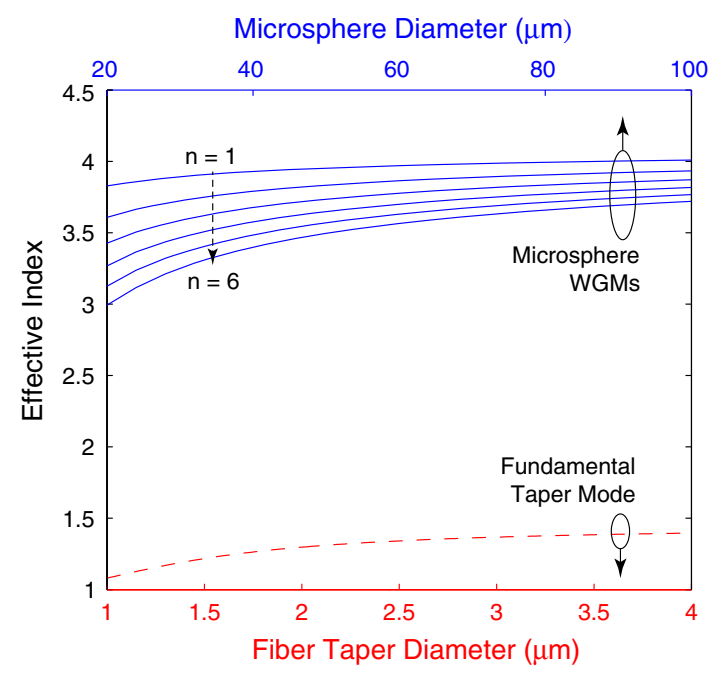

Fig. 5. (Color online) Effective index at $\lambda=2 \mu \mathrm{m}$ for the fundamental mode of a silica fiber taper with diameter $d$ in the range 1-4 $\mu \mathrm{m}$ (red dashed curve) and whispering gallery modes in a Ge microsphere for different sphere sizes (blue solid curves). The latter are calculated for radial mode numbers $1 \leq$ $n \leq 6$ and $m=l$, where $l$ and $m$ are the azimuthal and polar mode numbers; $l$ is chosen to provide resonances at $\sim 2 \mu \mathrm{m} 1$.

P. Wang is funded by the IRCSET, co-funded by the Marie-Curie Actions under FP7. G. Brambilla gratefully acknowledges the Royal Society (London) for his research fellowship.

\section{References}

1. D. Ahn, C.-Y. Hong, J. Liu, W. Giziewicz, M. Beals, L. C. Kimerling, J. Michel, J. Chen, and F. X. Kärtner, Opt. Express 15, 3916 (2007).

2. V. S. Ilchenko and A. B. Matsko, IEEE J. Sel. Top. Quantum Electron. 12, 15 (2006).

3. A. A. Savchenkov, V. S. Ilchenko, T. Handley, and L. Maleki, Opt. Lett. 28, 649 (2003).

4. A. A. Savchenkov, V. S. Ilchenko, T. Handley, and L. Maleki, IEEE Photon. Technol. Lett. 15, 543 (2003).

5. V. R. Almeida, C. A. Barrios, R. R. Panepucci, and M. Lipson, Nature 431, 1081 (2004).

6. Y. O. Yilmaz, A. Demir, A. Kurt, and A. Serpenguzel, IEEE Photon. Technol. Lett. 17, 1662 (2005).

7. N. Vukovic, N. Healy, P. Horak, J. R. Sparks, P. J. A. Sazio, J. V. Badding, and A. C. Peacock, Appl. Phys. Lett. 99, 031117 (2011).

8. S.-L. Cheng, G. Shambat, J. Lu, H.-Y. Yu, K. Saraswat, T. I. Kamins, J. Vuckovic, and Y. Nishi, Appl. Phys. Lett. 98, 211101 (2011).

9. A. Ouvrard, A. Garnache, L. Cerutti, F. Genty, and D. Romanini, IEEE Photon. Technol. Lett. 17, 2020 (2005).

10. J. Ballato, T. Hawkins, P. Foy, B. Yazgan-Kokuoz, R. Stolen, C. McMillen, N. K. Hon, B. Jalali, and R. Rice, Opt. Express 17, 8029 (2009).

11. G. R. Elliott, D. W. Hewak, G. S. Murugan, and J. S. Wilkinson, Opt. Express 15, 17542 (2007).

12. G. S. Murugan, Y. Panitchob, E. J. Tull, P. N. Bartlett, D. W. Hewak, M. N. Zervas, and J. S. Wilkinson, J. Appl. Phys. 107, 053105 (2010).

13. B. E. Little, J. P. Laine, and H. A. Haus, J. Lightwave Technol. 17, 704 (1999).

14. N. K. Hon, R. Soref, and B. Jalali, J. Appl. Phys. 110, 011301 (2011). 